\section{LA-UR- $07-1067$}

Approved for public release;

distribution is unlimited.

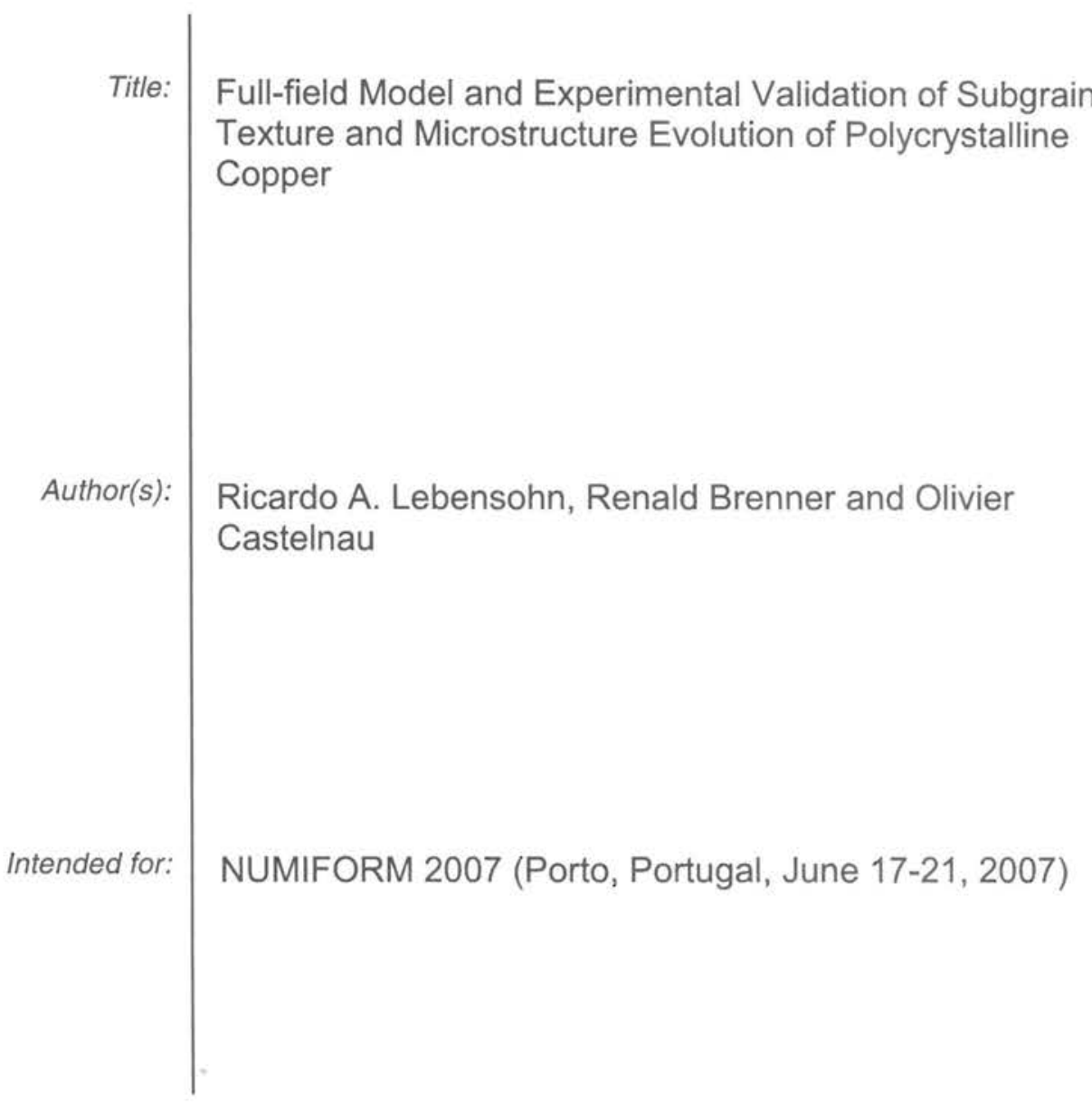

- Los Alamos

- EST.1943

Los Alamos National Laboratory, an affirmative action/equal opportunity employer, is operated by the Los Alamos National Security, LLC for the National Nuclear Security Administration of the U.S. Department of Energy under contract DE-AC52-06NA25396. By acceptance of this article, the publisher recognizes that the U.S. Government retains a nonexclusive, royalty-free license to publish or reproduce the published form of this contribution, or to allow others to do so, for U.S. Government purposes. Los Alamos National Laboratory requests that the publisher identify this article as work performed under the auspices of the U.S. Department of Energy. Los Alamos National Laboratory strongly supports academic freedom and a researcher's right to publish; as an institution, however, the Laboratory does not endorse the viewpoint of a publication or guarantee its technical correctness.

Form $836(7 / 06)$ 


\title{
Full-field Model and Experimental Validation of Subgrain Texture and Microstructure Evolution of Polycrystalline Copper
}

\author{
Ricardo A. Lebensohn ${ }^{1}$, Renald Brenner ${ }^{2}$ and Olivier Castelnau ${ }^{2}$ \\ (1) Materials Science and Technology Division, Los Alamos National Laboratory, Los Alamos, NM 87544, USA. \\ (2) Laboratoire des Proprietés Mécaniques et Thermodynamiques des Matériaux, Université Paris XIII, Av J.- \\ B.Clement, 93430 Villetaneuse, France.
}

\begin{abstract}
An efficient full-field formulation based on Fast Fourier Transforms (FFT) for the prediction of the viscoplastic deformation of polycrystals is applied to the study of the subgrain texture and microstructure evolution in a copper aggregate deformed under tension. Direct input from OIM images is used in the construction of the initial unit cell. Average orientations and misorientations predicted with the FFT-based approach after $11 \%$ tensile strain are directly compared with OIM measurements, showing a reasonable agreement. The differences between misorientations of surface grains compared with bulk grains are estimated, and the orientation-dependence of grain's misorientations is studied. Measurements and simulations agree in that grains with initial orientation near (110) tend to develop higher misorientations, as deformation proceeds. This behavior can be explained in terms of attraction towards the two different stable orientations and grain interaction. Only models that account for grain interaction, like the FFT-based formulation, are able to capture these effects.
\end{abstract}

Keywords: Texture, Microstructure, Polycrystal, Copper, Orientation Imaging Microscopy, Fast Fourier Transform, Micromechanical Fields.

\section{INTRODUCTION}

The computation of the mechanical behavior of polycrystalline materials based on their microstructure and the properties of their constituent single-crystal grains is a central problem in materials science. In recent years, the advances in the theories that link microstructures and properties, combined with the increasing numerical capability of computers, have enabled the development of new concepts and algorithms for predicting the effective response and the local statistics of polycrystals with a given microstructure, and the actual local mechanical fields that appear inside a polycrystal with a particular configuration, as well.

The finite element method (FEM) has been extensively applied to obtain full-field solutions for the plastic deformation of polycrystalline materials, with intracrystalline resolution. However, the difficulties related to meshing and the large number of degrees of freedom required by such FEM calculations limits the complexity and the size of the microstructures that can be investigated by these methods.
In this work we report on a new application of an alternative formulation, based on Fast Fourier Transform [1-4], which delivers a very detailed mapping of the intragranular mechanical fields that develop in a large polycyrstalline unit cell, in a very efficient fashion compared with a FEM calculation.

The novelty of the present application consists in the use of the electron back-scattering diffraction (EBSD) technique to obtain the actual digitalized microstructure of a polycrystal. In turn, this information is used to assign material properties to the points of a regular grid, as needed for a straightforward implementation of a FFT-based calculation for this particular microstructure. This approach, with direct input from a real microstructure, is used here to study the orientation dependence of the intragranular misorientations that develop in fcc materials under uniaxial tension.

\section{EXPERIMENTAL}

EBSD-based Orientation Imaging Microscopy (OIM) was used to characterize the local orientations 
on an area of about $500 \times 500$ microns, lying on one of the flat surfaces of a recrystallized copper sample, with a resolution (i.e. given by the distance between two consecutive pixels) of 2 microns in each direction. Two OIM images were taken, one from the undeformed sample, and another after $11 \%$ tensile strain at $20^{\circ} \mathrm{C}$, along the $y$-direction. The scanned area of the deformed sample (332x 445 pixels) was larger than the one of the initial microstructure $(274 \times 339$ pixels), and contained more orientations (2429 vs. 1585 grains). This allowed us to register both images, and therefore to identify the (distinct) indices given by the OIM software to individual grains, before and after deformation. The registering procedure was carried out by: a) identifying, by eye inspection of sizes and shapes (which did not change too much after $11 \%$ strain), the indices of the five largest grains, in both images, and b) optimizing the relative position of the initial (smaller) image inside the final (larger) image. For a given relative position of both images, every pair of overlapping pixels was analyzed. Each time the indices of the five largest grains in both images coincided, the "score" of the comparison was improved, while every disagreement was penalized. Once the two images were appropriately registered, a correlation table (based essentially in the determination of the pixel partition of every grain belonging to one image among the grains of the other image) allowed us to easily identify the indices of at least 20 of the largest grains, in the pre- and postdeformation images.

As part of the OIM package, it was possible to obtain the average orientation and the average misorientation associated to every one of the identified grains. After 11\% strain, the average over 2429 orientations of the per-grain average misorientation was $1.71 \mathrm{deg}$, with a maximum value of $6.62 \mathrm{deg}$. The accuracy of the average values of orientation and misorientation in the grains delivered by the built-in OIM software was confirmed by our own analysis (based on the quaternion representation of crystallographic orientations $[5,6])$, which was developed for the post-processing of the FFT-based results.

\section{MODEL}

The FFT-based full-field formulation for viscoplastic polycrystals is conceived for periodic unit cells, provides an exact solution of the governing equations, and has better numerical performance than a FE calculation for the same purpose and resolution. It was originally developed [1-2] as a fast algorithm to compute the elastic and elastoplastic effective and local response of composites, and later adapted [3-4] to deal with the viscoplastic deformation of threedimensional power-law polycrystals. Briefly, the viscoplastic FFT-based formulation consists in finding a strain rate field, associated with a kinematically admissible velocity field, which minimizes the average of local work rate, under the compatibility and equilibrium constraints. The method is based on the fact that the local mechanical response of a periodic heterogeneous medium can be calculated as a convolution integral between the Green function of a linear reference homogeneous medium and the actual heterogeneity field. Since such type of integrals reduces to a simple product in Fourier space, the FFT algorithm can be used to transform the heterogeneity field into Fourier space and, in turn, to get the mechanical fields by antitransforming that product back to real space. However, the actual heterogeneity field depends precisely on the a priori unknown mechanical fields. Therefore an iterative scheme should be implemented to obtain, upon convergence, a compatible strain-rate field and a stress field in equilibrium (see $[2,4]$ for details).

\section{UNIT CELL CONSTRUCTION}

A 2-D 256x256 image, containing information on the local (pixel by pixel) crystallographic orientation and a total of 1124 grains, was cropped from the original OIM image, obtained from the free surface of the undeformed $\mathrm{Cu}$ sample, consisting originally of $274 \times 339$ pixels and 1585 grains. The average grain size of the cropped image, given by: $\sqrt{256 \times 256 / 1124}=7.64$ pixels was very close the original value of $\sqrt{339 \times 274 / 1585}=7.66$ pixels. The actual 3-D microstructure of the bulk of the sample was not known. Therefore, a 3-D unit cell was built assuming a randomly-generated distribution of bulk grains beneath the measured surface grains (i.e. a "random substrate"), having the same average grain size as the one of the surface grains. For this, a 3-D Voronoi was generated (note that, since the FFT-based calculation requires a discrete description of the microstructure on a regularly-spaced grid, the procedure is simpler than in the case of having to determine the exact position of the boundaries between Voronoi cells in a continuum), as follows:

a) The number of Fourier points in the third dimension (z-direction) was chosen to be 32 , resulting in a unit cell of $256 \times 256 \times 32=2,097,152$ Fourier points. Note that this choice gives, in average, about 4 grains along third dimension.

b) The number of grains of the Voronoi structure was calculated as $2,097,152 /(7.64)^{3} \cong 4,703$. 
c) Then, 4,703 points were randomly distributed in a 3-D unit cell. This Poisson distribution of points constitutes the nuclei of the random grains.

d) The sides of the unit cell were divided into equispaced $256 \times 256 \times 32$ Fourier points, or voxels. Each Fourier point was assigned to its nearest nucleus (accounting for periodic boundary conditions across the unit cell limits), determining 4,703 different domains (grains).

Next, the measured 2-D and the numericallygenerated 3-D microstructures were merged as follows: every 3-D grain having a voxel on the first $z$ layer was removed, and every voxel corresponding to these removed grains was assigned with the crystallographic orientation of the pixels of the OIM image having the same $x$ - and $y$-coordinates.
After carrying out these replacements, the first layer of the resulting unit cell turned out to have the exact same topology as the OIM image. However, without any further manipulation of this initial configuration, the measured "surface" grains become bulk grains, upon the imposition of periodic boundary conditions across the unit cell. Therefore, in order to reproduce the actual free surface condition on the measured grains, the bottom five $z$-layers of Fourier points were replaced by a buffer zone, or "gas phase", with infinite compliance (i.e. identically zero local stress). The resulting new configuration of the 3-D unit cell, including the zero-stress buffer zone can be seen in Figure 1.

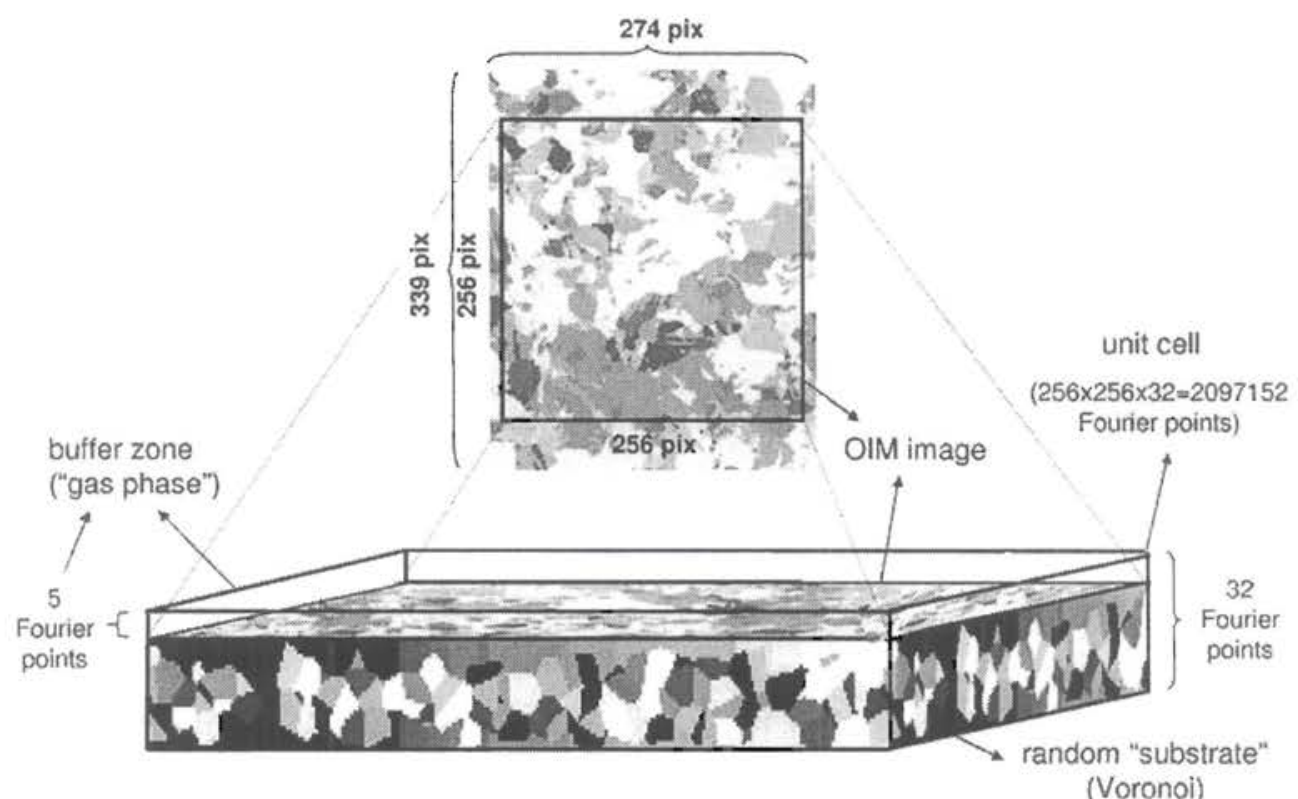

FIGURE 1. 3-D unit cell corresponding to the FFT-based simulations of local orientation and misorientation evolution, using direct input from OIM images.

In what follows, we show and compare results of both unit cell configurations, i.e. the original one resulting from the merging of the OIM and Voronoi structures, with no buffer zone, which amounted to neglect the surface character of the grains whose orientations were measured by OIM; and the one including the "gas phase", for a direct comparison with the OIM measurements.

It is worth noting that, unlike the above described Voronoi microstructure (in which periodic boundary conditions were imposed through the unit cell boundaries during its generation), the OIM image exhibits abrupt changes (from grains located inside the unit cell to other, completely decorrelated grains located across the unit cell limits) when periodic boundary conditions are imposed. This could deteriorate the model predictions, especially for grains close to the unit cell boundaries. However, the inaccuracies associated with the presence of these spurious "grain boundaries" seem to be of minor importance (see [7] for details). 


\section{RESULTS}

FFT-based simulations of the tensile deformation of the polycrystalline copper sample were performed using the two above-described unit cells (with and without "buffer zone"). A rate-sensitive crystal plasticity equation (with a viscoplastic exponent of 20) was used as local constitutive relation [3], assuming glide on $(111)<110>$ as the single active slip mode. The extended Voce law hardening parameters [8] used in the FFT-based calculation were adjusted to match the experimental macroscopic stress-strain curve measured during the tensile deformation of the copper sample (see [9] for details).
Figure $2 \mathrm{a}$ shows the registered initial and $11 \%$ strain OIM images (the latter is already cropped), measured on the surface of the copper sample. The post-deformation image clearly indicates the development of intragranular misorientations, in terms of noticeable color grades inside several grains.

The location and number of pixels of some of the largest ("marked") grains are shown in Figure 2b. Grains \#1 to \#5 are the five largest grains (in that order). The size of grain \#13 ranks 13th overall, but it is the largest one near the (110) orientation. The orientations of grains \#1 to \#5 and \#13 can be seen in an inverse pole figure representation (i.e. tensile axis in crystallographic axes), in Figure $2 c$.
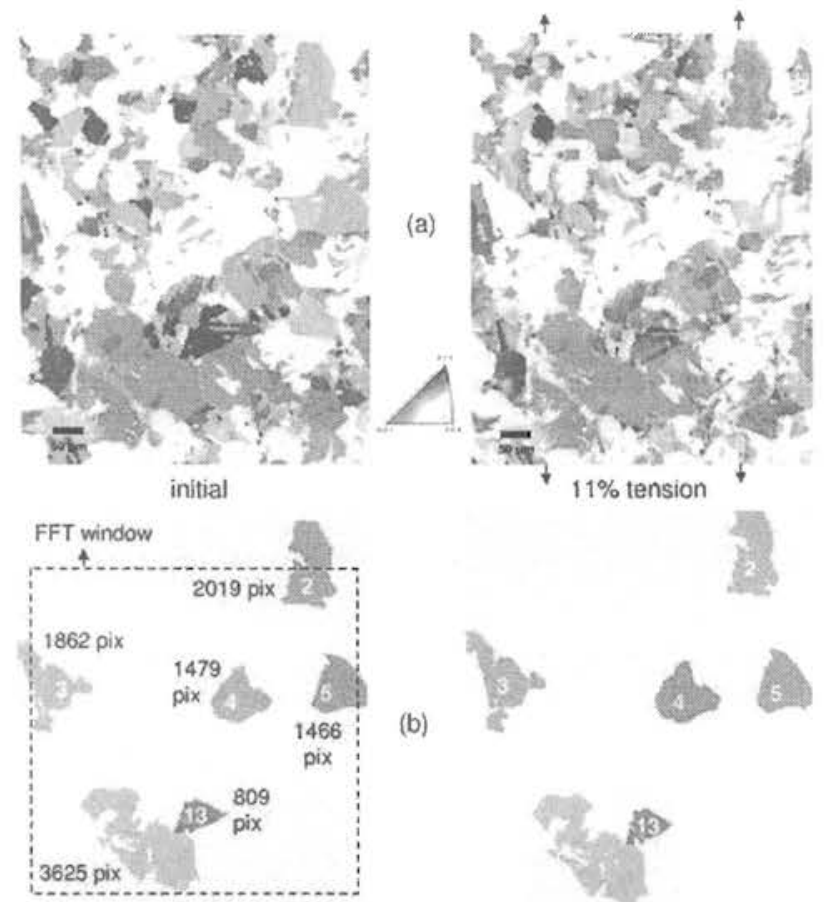

(a)
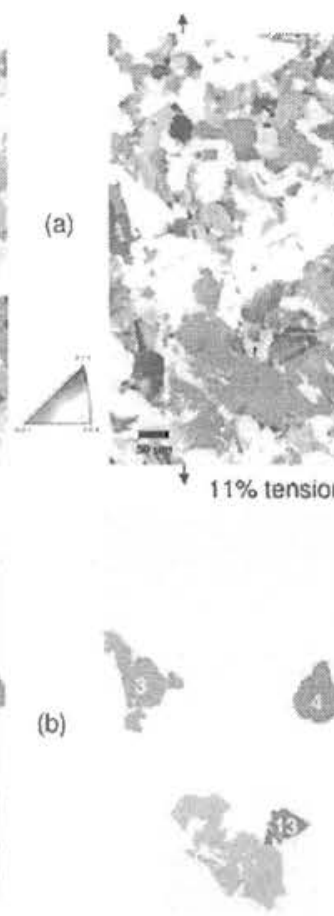

\begin{tabular}{|l|l|l|l|}
\hline $\begin{array}{c}\text { gr } \\
\text { i }\end{array}$ & OIM & FFT & $\begin{array}{c}\text { FFT no } \\
\text { buffer }\end{array}$ \\
\hline 1 & 2.89 & 2.41 & 1.78 \\
\hline 2 & 2.52 & 2.70 & 1.94 \\
\hline 3 & 2.92 & 2.89 & 1.98 \\
\hline 4 & 2.86 & 2.34 & 2.10 \\
\hline 5 & 2.26 & 2.68 & 1.93 \\
\hline 13 & 3.09 & 3.37 & 2.49 \\
\hline
\end{tabular}

(d) average misorientations [deg]
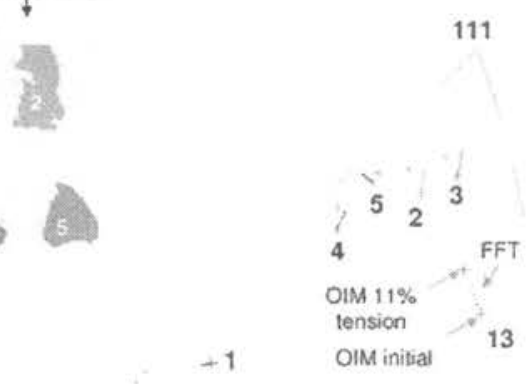

001

(c) average orientations

110

FIGURE 1. (a) Registered OIM images of the copper polycrystal before deformation and after $11 \%$ tensile strain. (b) Location and number of pixels of 6 large grains, before and after $11 \%$ strain. (c) Inverse pole figure of the measured initial and final average orientation of the 6 large grains, and trajectories predicted with the FFT-based approach. (d) Table showing the average misorientations of the 6 large grains after $11 \%$ tensile strain, measured and predicted with the FFT-based approach, with and without buffer zone.

Figure $2 \mathrm{c}$ also shows the trajectories of the mean orientations of the "marked" grains, as predicted by the FFT-based model. The small crosses defining these trajectories were obtained in increments of $1 \%$ overall strain. The actual final average orientations, measured with OIM, are shown as well. While grain \#1 rotates towards the stable orientation (001), all the others are attracted towards the other stable orientation, i.e. (111). The total rotation of the near-(110) grain (\#13) is the largest. The predicted reorientation of grain \#13 
matches almost exactly with the experiments, while the rotations of the rest of the "marked" grains show qualitative agreement, with maybe the exception of grain \#5.

The above-described reasonable agreement between the measured and predicted average orientation evolution is not surprising, since almost every (either full-field or mean-field) model based on crystal plasticity predicts the development of two stable texture components in (100) and (111) in fcc materials deformed under tension. A much less investigated aspect of the texture evolution of these materials is reported in Figure 2d. The table therein shows the comparison between the measured and the predicted values of average misorientations (in degrees) inside the "marked" grains. Two predicted values are reported for each grain: the third column shows the predictions obtained using the unit cell shown in Fig. 1, i.e. including the buffer zone, therefore considering the effect of measuring misorientations in surface grains. The fourth column displays the predictions obtained for an unit cell without buffer zone, giving an idea of the misorientations values that would be measured if the grains were bulk grains.

Evidently, the proper consideration in the model of the surface character of the grains whose average misorientations were measured by OIM, leads to a very reasonable matching with the corresponding experimental values. On the other hand, the artificial assumption of the bulk character of these grains, tends to underestimate (by a factor of about 0.75 ) the actual average misorientations of surface grains. The reason why the predictions obtained under the bulk assumption fall short is related with a more constraint configuration, compared with the actual boundary conditions imposed on the surface grains.
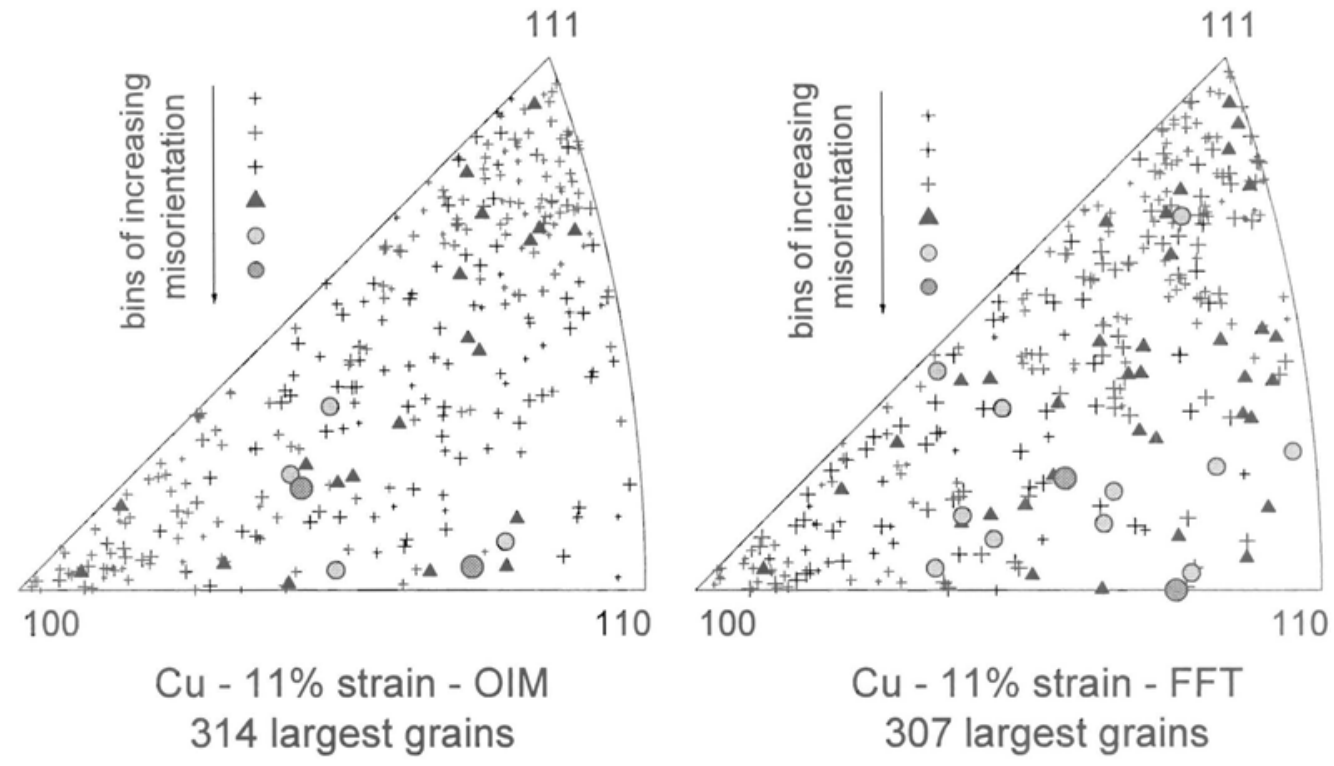

FIGURE 3. Inverse pole figures of the average orientations and misorientations of the $\sim 300$ largest grains, after $11 \%$ tensile strain, (a) measured by OIM, and (b) predicted with the FFT-based approach. The misorientations were grouped in bins of equal size, and different symbols were assigned to each bin.

Another interesting observation is that the grain near (110) (\#13) exhibits the largest average misorientation among the "marked" grains. This could be related to some kind of systematic orientation dependence of the average misorientation, or just be a local effect, related with the particular environment of grain \#13.

To elucidate this, we investigated the behavior of a larger number of representative grains. Figure 3 shows the average orientations (given by each projection in the inverse pole figure) and the average misorientations (given by the different symbols used) of the largest $\sim 300$ grains, as measured by OIM and predicted with the FFT-based approach, after $11 \%$ tensile strain. The misorientation values of the grains were grouped into six bins of equal size, and different symbols were assigned to each bin. 
The first observation is that after $11 \%$ tension there is already a mild but noticeable trend of the grains to rotate towards one of the stable (100) and (111) orientations. Moreover, it is evident (from both the experiments and the simulations) that most of the grains with the highest average misorientation are grains transitioning from their initial orientation near (110) towards the stable orientations. This observation can be explained in the following terms: depending on their initial orientation, the grains of an fcc polycrystal in tension are attracted towards one of the two stable orientations, i.e. (100) or (111). Grains with orientations in a region of the orientation space, spanning from near (110) to a turning point located somewhere between (100) and (111), are simultaneously "pulled" towards both stable orientations. In this case, the interaction with neighbor grains may define the preference of different portions of these "indecisive" grains to rotate towards different stable orientations. This conflicting attraction towards two completely different orientations may be accommodated by the development of relative higher misorientations between different grain's sub-domains.

\section{CONCLUSIONS}

The FFT-based full-field formulation for viscoplastic polycrystals was applied to the study of the subgrain texture evolution in a copper aggregate deformed under tension. Direct input was obtained from OIM images, for the construction of the initial unit cell. A methodology to build a three-dimensional unit cell, including the 2-D OIM data, a 3-D random substrate, and the presence of a free surface was given. The average orientations and misorientations of large grains, predicted with the FFT-based approach after $11 \%$ tensile strain, were directly compared with OIM measurements. The experimental data and the predictions showed a reasonable agreement. The differences of having measured misorientations in surface grains, compared with bulk grains was estimated. A ratio of about 0.75 between average misorientations developing inside bulk and surface grains was obtained. The orientation-dependence of the average misorientations was also studied. Again, measurements and predictions showed reasonable agreement. Grains with initial orientation near (110) tend to develop higher misorientations, as deformation proceeds. Attraction towards the two different stable orientations (i.e. corresponding to the alignment of the (100) and the (111) crystal orientations with the tensile axis) of different subdomains inside these grains, influenced by interactions with different neighbors, may be responsible for this behavior. Only models like the FFT-based formulation, which accounts for topologic information and grain interaction in the determination of the local micromechanical fields, may be able to capture these effects.

\section{REFERENCES}

1. H. Moulinec and P Suquet, Comput. Meth. Appl. Mech. Eng. 15, 69-94 (1998).

2. J.C. Michel, H. Moulinec and P. Suquet, Comput. Model. Eng. Sci. (CMES) 1, 79-88 (2000).

3. R.A. Lebensohn, Acta Mater. 49, 2723-2737 (2001)

4. R.A. Lebensohn, Y. Liu and P. Ponte Castañeda, Acta Mater. 52, 5347-5361 (2004).

5. A. Morawiec, J. Appl. Cryst., 31, 818-819 (1998).

6. J.C. Glez and J. Driver, J. Appl. Cryst. 34, 280-288 (2001).

7. R.A. Lebensohn, O. Castelnau, R. Brenner and P Gilormini, Int. J. Solids Struct. 42, 5441-5459 (2005).

8. C.N. Tomé, G.R. Canova, U.F. Kocks, N. Christodoulou and J.J. Jonas, Acta Metall. 32, 1637-1653 (1984).

9. R.A. Lebensohn, O. Castelnau and R. Brenner, in preparation. 